\title{
Time Series Analysis Of Monthly Sales Of Petroleum Products (A Case Study Of Nigerian National Petroleum Corporation, Nnpc - Enugu, From 1996 - 2003)
}

\author{
Osanebi Fidelis, And Obuba Okeoghene \\ Mathematics And Statistics Department Delta State Polytechnic, P.M.B 5 Ozoro, Delta State, Nigeria
}

\begin{abstract}
Time series is the name given to the values of some statistical variables measured over a uniform set of time points which may represent the historical performance of some economic or business variable. Examples of time series are total monthly sales receipts in a departmental store, total monthly sales of petroleum products, total monthly production by company, and consumption of electricity in kilowatts data on population motor registration. This research work is focused on investigating the monthly sales of petroleum products by Nigerian National Petroleum Corporation for the past eight years (1996 to 2003) and to construct an autoregressive model of a suitable order for the process.
\end{abstract}

Keyword: - Electricity, Time series Model, Stochastic Model, Crude oil, Economy

\section{INTRODUCTION}

The search of oil in Nigeria started as early as 1937, but the discovery was not until 1956. The sole of petroleum products began in December 1957, managed by a consortium of Royal Dutch Shell and British Petroleum BP Now known as Shell Petroleum Development Company SPDC.

G.A, Aga (1993) stated that Nigeria was the second oil producing nation in Africa after Libya and sixth in the world. In May 1971, the Nigeria National Oil Company was established under the company and Allied matter Act of 1958 as applicable then. NNOC was the government Agency Mandated by law to engage in all phases of oil production and sales, NNOC was later in 1977 amalgamated into a full flex ministry of petroleum to form the Nigeria National Petroleum Corporation (NNPC), which is in partnership with several oil company from different countries operating in Nigeria. Before October 1965, Nigeria Crude Oil was refined overseas and all the processed oil needs were imported. The first refinery plant came into operation in 1965 located at Alesa Eleme near Port-Harcourt. Later Warri and Kaduna Petro-chemical refineries were established in 1978 and 1980 respectively. Similarly, Pipeline and Products Marketing Company Ltd (PPMC) Enugu Depot was commissioned in 25 August 1975 by the then military Governor of the old Anambra State; Colonel D.S. Abubakar.

The last was the second refinery in Port-Harcourt. It is however worthy to note that NNPC has several subsidiary company e.g. Pipeline and Product Marketing Company (PPMC).

\section{II. NIGERIAN NATIONAL PETROLEUM CORPORATION (ITS ROLE IN SALE OF PETROLEUM PRODUCTS)}

The NNPC's role in Oil Industry is so much that it cannot handled it alone. This is the reason for the establishment of subsidiary company like pipeline and Products Marketing Company Ltd (PPMC).

The Nigerian National Petroleum Corporation manages the affairs of the oil industry in Nigeria, while the PPMC under the corporation is in charge of sales of petroleum products.

Government policy on oil matter such sales is been conveyed by the Petroleum Products Price Regulatory Agency (PPPRA) currently headed by Alhaji Gbalamosi. NNPC therefore, works in conjunction with PPPRA to implement government policy such as prices of petroleum products. Nigerian National Petroleum Corporation carries out its function as such in both local and international.

\section{STATEMENT OF PROBLEM}

Over the years, the frequent review of prices of petroleum products has gain a space in the heart of Nigerians, and most of the time, the out of stock of petroleum products at depot for sales is also rampart. In view of the above statement, the project examines total monthly sales of petroleum products in Nigeria National Petroleum Corporation in Enugu State and build a stochastic model for the data obtained.

\section{OBJECTIVES OF STUDY}

1. To determine the stationarity of sales of petroleum products. 
2. To construct an autoregressive model of a suitable order for the process.

3. To forecast the series for sales in 2004 quarters.

4. To make recommendation based on the findings of the research

\section{LITERATURE REVIEW}

In view of that fact that it is necessary to upgrade standard in other to meet the text of time and improved models on ground. The researcher is poised to consolidate on the work done by some researchers in the past on related topics. This research work therefore, reviews the works of past researchers and their reports as contained in textbooks, Newspapers, Bulletin and Journals on sales of petroleum products in Nigeria.

Prof Jubril Aminu, Hon Minister of Petroleum Resources (1990) saw the sales of petroleum products as a function of production since research is abundant. He emphasized that investment has been low in all OPEC nations and Nigeria in general. He stress that unless we increase our investment, production would decline and this would adversely affect sales.

Dr. T.M John (1990) in his speech said that "there is too much waste in NNPC, the management style and habits are most wasteful". He emphasized that waste abounds in NNPC namely at the plants, in projects and in support services. He said in NNPC we replace rather than maintain and repair, we buy in excess of our requirement at prices higher than commercial average and from source capabilities lower than commercial standard.

The society's view is that the general purpose of sales of petroleum products is to add comfort to the wellbeing of mankind. The way to fulfill this purpose is to produce meaningful work for the members of the society as well to distribute adequate oil and services to the needs of member of the society.

Unfortunately, government policy of the day seems to be inimical to this concept. The frequent increases in prices of petroleum product over the year has not address the plight of the masses. It is however hoped that economic reform program embarked upon by the present administration of President Olusegun Obasanjo will meet the need and yearning of the people

\section{METHODOLOGY}

This research highlights the methods used in analyzing the data, and they are:

\section{AUTOCORRELATION FUNCTION}

Considering the fact that it is usually impossible to obtain a complete description of a stochastic process (i.e. actually specifying probability distribution), the autocorrelation function provides a particular description of the process for modeling purpose. The autocorrelation function discusses how much correlation there is or how much interdependency there is between neighboring data point in the series $y_{t}$

We define the Autocorrelation with lag $\mathrm{k}$ as

$$
\begin{aligned}
p_{k}= & \frac{E\left(\left(y-y_{t}\right)\left(y_{t+k}-y_{t}\right)\right.}{\sqrt{E\left(y-y_{t}\right)^{2} E\left(y_{t+k}-y_{t}\right)^{2}}} \\
= & \frac{\operatorname{cov}\left(y_{t} y_{t+k}\right)}{\sigma_{y} \sigma_{y+k}}
\end{aligned}
$$

For a stationary process, the variance at time $\mathrm{t}$ is the same as the variance at time $t+k$ hence, the denominator is just the variance of the stochastic process and

$$
p_{k}=\frac{E\left(\left(y-y_{t}\right)\left(y_{t+k}-y_{t}\right)\right.}{\sigma_{y} \sigma_{y+k}}
$$

Thus,

$$
\text { The numerator is the covariance between } y_{t} \text { and } y_{t+k}, \Upsilon_{\mathrm{k}}
$$

$$
\mathrm{P}_{\mathrm{k}}=\Upsilon_{\mathrm{k}} \Rightarrow \mathrm{Po} \quad=\Upsilon_{0}=1 \text { for any stochastic process }
$$

Suppose that the stochastic process is $y_{t}=\sum_{\mathrm{t}}$

Where $\sum_{\mathrm{t}}$ is an independently distributed random variable with zero mean.

The autocorrelation function for this process given is called a White Noise and there is no model that can provide a forecast any better than $y_{t+l}=0$ for all $\mathrm{L}$

Thus if the autocorrelation function $\mathrm{P}_{\mathrm{k}}$ is zero (or close to zero) for all $\mathrm{K}>0$, there is little or no value in using a model to forecast the series.

The autocorrelation function $\mathrm{P}_{\mathrm{k}}$ is theoretical and in practice, an estimate of the autocorrelation function called the sample autocorrelation function. 


$$
p_{k}=\frac{\sum_{t=1}\left(y-y_{t}\right)\left(y_{t+k}-y_{k}\right)}{\sum_{t=1}^{t-k}\left(y-y_{t}\right)^{2}}
$$

\section{AUTOREGRESSIVE MODELS}

The autoregressive process of order $\mathrm{P}$ in the current observation. It is generated by a weighted average of Past observations going back $\mathrm{P}$ periods, together with a random in the current period denoted by AR (P) and with equation as:

$$
y_{t}=\quad \phi_{1} y_{t-1}+\phi_{2} y_{t-2}+\ldots+\phi_{\mathrm{p}} y_{t-p}+\delta+\sum_{\mathrm{t}}
$$

Where $\delta$ Is a constant term which relates to the mean of the stochastic process?

\section{NOTE}

If the autoregressive process is stationary, then its mean is denoted by $\mu$ which must be invariant with respect to time. i.e.

$$
\sum\left(y_{t}\right)=\sum\left(y_{t-1}\right)=\sum\left(y_{t-2}\right)=, \ldots, \mu
$$

Which can also be given by:

$\mu=\phi_{1} \mu+\phi_{2} \mu+\ldots+\phi_{p} \mu+\delta$

Or $\mu=-\phi_{1}-\phi_{2}-\ldots-\phi_{\mathrm{p}}$

The mean of the process also gives the stationarity such that the process is stationary, the mean $\mu$ must be finite and so

$\phi_{1}+\phi_{2}+\ldots+\phi_{p}<1$

To examine the properties of a simple autoregressive process we must determine the mean, variance and covariance for the process i.e. first order autoregressive process AR (I)

$$
y_{t}=\phi_{1} y_{t-1}+\delta+\sum_{t}
$$

This process has the mean

$$
\mathrm{M}=\frac{\delta}{1-\emptyset_{1}} \text { Stationary if }|\emptyset|<1
$$

The variance $\Upsilon_{0}$ about its means, assuming stationary and setting $\delta=0$ i.e.

$$
\Upsilon_{0}=\frac{\sigma \Sigma^{2}}{1-\emptyset_{1}{ }^{2}}
$$

The covariance of $y_{t}$ about the mean is

$\Upsilon_{1}=\frac{\sum\left(y_{t-1}\left(\phi y_{t-1}+\Sigma_{t}\right)\right.}{\phi 1 \Upsilon 0}$

$\Upsilon_{1}=\frac{\sigma \Sigma^{2}}{1-\phi_{1}{ }^{2}}$

\section{NOTE}

The autocorrelation function for AR (I) begins at $\mathrm{P}_{0}=1$ and declines geometrically

$$
p_{k}=\frac{\Upsilon_{k}}{\Upsilon_{0}}=\phi_{1}{ }^{k}
$$

The second order autoregressive process AR (2)

$$
y_{t}=\phi y_{t-1}+\Upsilon \phi_{2} y_{t-2}+\sigma+\sum
$$

The process has mean

$\mu=\frac{\sigma}{1-\Upsilon \phi 1-\phi 2}$

And a necessary condition for stationary is that:

$\phi_{1}+\Upsilon \phi_{2}<1$

The variance and covariance of $y_{t}$ is measured in deviation form. That is:

$$
\begin{gathered}
\Upsilon_{0}=\sum\left(y_{t}\left(\phi_{1} y_{t-1}+\phi_{2} y_{t-2}+\sum_{\mathrm{t}}\right)\right) \\
=\phi_{1} \Upsilon_{1}+\phi_{2} \Upsilon_{2}+\sigma_{\Sigma}{ }^{2} \\
\Upsilon_{1}=\sum\left(y_{t-1}\left(\phi_{1} y_{t-1}+\phi_{2} y_{t-2}+\Sigma_{\mathrm{t}}\right)\right) \\
=\phi_{1} \Upsilon_{0}+\phi_{2} \Upsilon_{1} \\
\Upsilon_{2}=\sum\left(y_{t-2}\left(\phi_{1} y_{t-1}+\phi_{2} y_{t-2}+\sum_{t}\right)\right) \\
=\quad \phi_{1} \Upsilon_{1}+\phi_{2} \Upsilon_{0}
\end{gathered}
$$

And in general for $\mathrm{k} \geq 2$

$\Upsilon_{\mathrm{k}}=\sum\left(y_{t-k}\left(\phi_{1} y_{t-1}+\phi_{2} y_{t-2}+\sum_{\mathrm{t}}\right)\right)$ 


$$
=\phi_{1} \Upsilon_{\mathrm{k}-1}+\phi_{2} \Upsilon_{0}
$$

These equations can be solved simultaneously to get $\Upsilon_{0}$ in term of $\phi_{1}, \phi_{2}$ and $\sigma_{\Sigma}^{2}$ such that

$$
\Upsilon_{1}=\frac{\phi \Upsilon_{2}}{1-\phi_{2}}
$$

NOTE

These equation can be used to derived the autocorrelation function $P_{k}$

$\mathrm{P}_{1}=$

$$
\begin{aligned}
& \frac{\phi_{1}}{1-\phi_{2}} \cdots \\
& \text { (1) } \quad p_{2}=\underline{\Upsilon \phi_{2}} \underline{+} \underline{\phi_{1}}{ }^{2} \\
& 1-\phi_{2}
\end{aligned}
$$

For $\mathrm{K} \geq 2, \quad \mathrm{P}_{\mathrm{k}}=\Upsilon \phi_{1} \mathrm{P}_{\mathrm{k}-1}+\Upsilon \phi_{2} \mathrm{P}_{\mathrm{k}-2}$ which can be used to calculate autocorrelation function for $\mathrm{K}>2$

Equation (1) and (2) are called the Yule Walker equations. Suppose we know the sample autocorrelation functions for a time Series that is second - order Autoregressive process or more i.e. $\mathrm{P}_{1}$ and $\mathrm{P}_{2}$ or $\mathrm{P}_{\mathrm{k}}$ could be measured and substituted in the Yule - Walker equation and solved simultaneously for the unknown $\phi_{1}$ and $\phi_{2}$ or $\phi_{\mathrm{k}}$.

The essence of Yule - Walker equations implies therefore, that they could be used to obtain estimates of the Autoregressive parameters $\phi_{1}$ and $\phi_{2}$ or $\phi_{\mathrm{k}}$.

\section{PARTIAL AUTOCORRELATION FUNCTION P.A.C.F}

The partial autocorrelation function is denoted by $\left(\Upsilon \phi_{\mathrm{kk}}: \mathrm{K}=1,2 \ldots\right)$ the set of partial autocorrelation at various lags $\mathrm{K}$ are defined by.

Where

$$
\phi_{\mathrm{Kk}}=\frac{\left(p_{k}{ }^{*}\right)}{p_{k}}
$$

$\mathrm{P}_{\mathrm{k}}$ is the $\mathrm{kx} \mathrm{k}$ autocorrelation matrix and $\mathrm{P}_{\mathrm{k}}{ }^{\mathrm{x}}$ is $\mathrm{P}_{\mathrm{k}}$ with the last column replaced by

$\mathrm{P}_{1}$

$$
\begin{aligned}
& \text { So } \quad\left(\begin{array}{l}
\dot{\mathrm{P}}_{\mathrm{k}} \\
\phi_{11}=\mathrm{P} \\
\phi_{22}
\end{array}\right)=\frac{\left[\begin{array}{ll}
1 & p_{1} \\
p_{1} & p_{2}
\end{array}\right]}{\left[\begin{array}{ll}
1 & p_{1} \\
p_{1} & 1
\end{array}\right]} \\
& =\frac{p_{2}-p^{2}}{1-p_{1}{ }^{2}}
\end{aligned}
$$

And an estimate $\phi_{\mathrm{kk} \text { can }}$ be obtained by replacing the $\mathrm{P}_{\mathrm{i}}$ by $\Upsilon_{1}$. At lags large enough for the p.a.c.f to have died out Quenouille's formula gives.

$$
\begin{aligned}
& \operatorname{Var}\left(\phi_{\mathrm{kk}}\right) \cong \frac{1}{N} \\
& \text { i.e. }\left(\phi_{\mathrm{kk}}\right) \cong \frac{1}{\sqrt{N}} \\
& \phi_{33}=\left[\begin{array}{ccc}
1 & p_{1} & 1 \\
p_{1} & 1 & p_{2} \\
p_{2} & p_{1} & 3_{3}
\end{array}\right] \\
& \hline\left[\begin{array}{ccc}
1 & p_{1} & p_{2} \\
p_{1} & 1 & p_{1} \\
p_{2} & p_{1} & 1
\end{array}\right]
\end{aligned}
$$

Obtain the determinant of the matrix above.

Under the hypothesis of $\Upsilon \phi_{\mathrm{kk}}=0, \mathrm{~K}=1,2 \ldots$ Quenoullis formular for $\mathrm{K}>1$ distribution with mean 0 and standard deviation $\frac{1}{T}$ where, $(\mathrm{T} \Rightarrow$ no of

Observations in the series)

Bartlett's formular provides approximately standard errors for the autocorrelations, so that the order of the moving average process can be determined from significant tests on the sample autocorrelations.

For the order of an autoregressive process, this can be obtained from the partial autocorrelation function. To understand what the partial autocorrelations functions is and how it can be used, let us consider the covariance and autocorrelation function for the autoregressive process of order $\mathrm{P}$. 
NOTE: The covariance with displacement $\mathrm{K}$ is determine from

$\Upsilon_{\mathrm{k}}=\sum\left(y_{t-k}\left(\phi_{1} y_{t-1}+\phi_{2} y_{t-2}+\ldots \ldots+\Upsilon \phi_{\mathrm{p}} y_{t-p}+\sum_{\mathrm{t}}\right)\right)$

Letting $\mathrm{K}=0,1 \ldots \mathrm{P}$, we obtain the following $\mathrm{P}+1$ differences equations that can be solved simultaneously for;

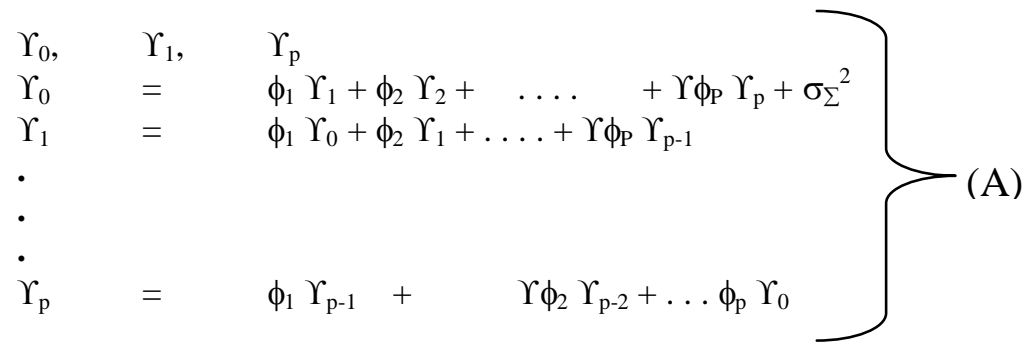

Autocorrelations $P_{1}=\phi_{1}$. Thus, if the calculated value of $\Upsilon \phi_{1}$ is significantly different from zero, then the autoregressive process is at least order I, and we denote this value $\phi_{1}=\mathrm{a}_{1}$.

Similarly, the hypothesis that $\mathrm{P}=2$, we solve the Yule - Walker equations for $\mathrm{P}=2$ gives us a new set of estimates $\phi_{1}$ and $\phi_{2}$. If $\phi_{2}$ is significantly different firm zero as well, we therefore conclude the process is at least order 2 , and $\mathrm{P}=2$ is denoted by $\phi_{2}$ as $\mathrm{a}_{2}$.

This process can be repeated successive values $P$ for $P=3$, obtained and estimate of $\Upsilon \phi_{3}$ denoted by $a_{3}$. The new sets of sequence $a_{1}, a_{2}, a_{3}, \ldots$ are called the partial autocorrelation functions and can be used to infer the order of the autoregressive process for its behaviour. In particular, if the true order of the process is $\mathrm{P}$, we should observe that $\mathrm{aj}=0$ for $\mathrm{j}>\mathrm{p}$.

For displacement to be greater than $\mathrm{P}$, the covariance are determine from:

$$
\Upsilon_{\mathrm{k}}=\phi_{1} \Upsilon_{\mathrm{p}-1}+\phi_{2} \Upsilon_{\mathrm{k}-2}+\ldots+\Upsilon \phi_{\mathrm{p}} \Upsilon_{\mathrm{k}-\mathrm{p}}
$$

Now dividing both sides in (A) by $\Upsilon_{0}$, we obtain a set of $\mathrm{P}$ equations that together determine the first $\mathrm{P}$ values of the autocorrelation function.

Ie.

$$
\begin{aligned}
& \mathrm{P}_{1} \quad=\quad \phi_{1}+\phi_{2} \mathrm{P}_{1}+\ldots+\phi_{\mathrm{p}} \mathrm{P}_{\mathrm{p}-1} \\
& \text {. } \\
& \text {. } \\
& \dot{\mathrm{P}}_{\mathrm{p}} \quad=\phi_{1} p_{p-1}+\phi_{2} p_{p-2}+\ldots+\phi_{\mathrm{p}}
\end{aligned}
$$

\section{NOTE}

Equations (B) are the Yule - Walker equations:

If $\mathrm{P}_{1}, \mathrm{P}_{2} \ldots \mathrm{P}_{\mathrm{p}}$ are known, then the equations can be solved for $\phi_{1}, \phi_{2} \ldots \phi_{\mathrm{p}}$.

Suppose we start by hypothesizing that $\mathrm{P}=1$. The equations (B) boils down to $\mathrm{P}_{1}=\phi_{1}$, or using the sample.

\section{FORECASTING: USING AN AUTOREGRESSIVE MODEL}

Having determine the model in which the chance element plays a dominant role in determining the structure of the model. Consider the simplest form of the Autoregressive model.

OR

$$
y_{t}=\Upsilon \phi_{1} y_{t-1}+\sum_{\mathrm{t}}
$$

The general form of $p t h$ order of the autoregressive process is defined by $y_{t}-\phi_{1} y_{t-1}-\phi_{2} y_{t-2}-\ldots \phi_{\mathrm{p}} y_{t-p}+\sum_{\mathrm{t}}$

Where $\phi_{1}, \phi_{2} \quad \ldots \phi_{\mathrm{p}}$ are constants and the model is denoted as AR (P) generally the model may be written as

Where

$$
y_{t}=\Upsilon \phi_{1} y_{t-1}+\sigma+\sum_{\mathrm{t}}
$$

$\sigma$ And $\sum_{t}$ are assume to be zero for a stationary time series.

\section{NOTE}

$\Upsilon \phi_{1}, \Upsilon \phi_{2}$ are obtained from - Yule - Walker equations

$\phi_{1}+\phi_{2} \mathrm{P}_{1}=\mathrm{P}_{1}$

$\phi_{1} \mathrm{P}_{1}+\phi_{2}=\mathrm{P}_{2}$

And the partial autocorrelation functions are obtained as $\phi_{1}=\mathrm{P}_{1}$ 


$$
\begin{aligned}
& \begin{aligned}
& \phi_{2}=\left.\frac{\left.\left[\begin{array}{cc}
1 & p_{1} \\
p_{1} & p_{2}
\end{array}\right)\right]}{\left[\begin{array}{cc}
1 & p_{1} \\
p_{1} & 1
\end{array}\right)}\right] \\
&= \underline{\mathrm{P}}_{2}-\mathbf{P}_{1}^{2} \\
& 1-\underline{\mathbf{P}}_{1}^{2}
\end{aligned} \\
& \phi_{2} \quad=0, \quad \mathrm{~K}>1
\end{aligned}
$$

$\operatorname{Var}\left(\phi_{\mathrm{kk}}\right) \cong \frac{1}{N}$

$\left(\phi_{\mathrm{kk}}\right) \cong \frac{1}{\sqrt{N}}$

The forecast for the simplest case is obtained as

$$
y_{t+1}=\phi_{1} y_{t}
$$

Similarly the future value $y_{t+2}$ can be obtain as follows

$$
\begin{gathered}
y_{t+2}=\phi_{1} y_{t+1} \\
y_{t+2}=\phi^{2} y_{t}
\end{gathered}
$$

\begin{tabular}{|c|c|c|c|c|c|c|c|c|}
\hline Month & 1996 & 1997 & 1998 & 1999 & 2000 & 2001 & 2002 & 2003 \\
\hline JAN & 60711101 & 65501003 & 62779811 & 63576009 & 68380201 & 61755364 & 67437076 & 72741486 \\
\hline FEB & 58814721 & 60113216 & 58003462 & 58449975 & 60435178 & 55781770 & 643240661 & 66142083 \\
\hline MAR & 60040001 & 63223741 & 62562201 & 63483375 & 64186064 & 58870795 & 68642359 & 70365536 \\
\hline APR & 63202613 & 61148848 & 60014841 & 59098118 & 60975796 & 61578707 & 65784376 & 72775451 \\
\hline MAY & 61746114 & 62883721 & 62983112 & 62634888 & 63760263 & 64797113 & 68815832 & 72612419 \\
\hline$\pi N$ & 58349101 & 60245717 & 61421141 & 59651963 & 61310173 & 59202669 & 66060544 & 72554396 \\
\hline$\pi$ & 62455100 & 60731113 & 59984002 & 63380117 & 59558565 & 61409897 & 67625754 & 70958795 \\
\hline AUG & 60848233 & 59123014 & 62343737 & 62967150 & 48871749 & 62968852 & 69925800 & 71234915 \\
\hline SEP & 60241155 & 62356018 & 60375241 & 64317483 & 59729366 & 62033098 & 67520656 & 72329038 \\
\hline $\mathrm{OCT}$ & 61589134 & 63245771 & 59994124 & 62465230 & 64654576 & 64778848 & 70179284 & 74688993 \\
\hline NOV & 58436717 & 60214551 & 61435071 & 63784366 & 59554104 & 63666618 & 69830308 & 71235804 \\
\hline $\mathrm{DEC}$ & 65343710 & 64324118 & 66315211 & 67423414 & 61164526 & 66662696 & 72828313 & 71711536 \\
\hline TOTAL & 73177700 & 74311083 & 738210236 & 751272066 & 732380561 & 743505427 & 808891963 & 85936452 \\
\hline
\end{tabular}

Thus, the general form is

$$
y_{t+h}=\Upsilon \phi_{1}{ }^{\mathrm{h}} y_{t}
$$

\begin{tabular}{|c|c|c|c|c|c|c|c|c|}
\hline MONTH & 1996 & 1997 & 1998 & 1999 & 2000 & 2001 & 2002 & 2003 \\
\hline JAN & 60.71 & 65.5 & 62.78 & 63.58 & 68.38 & 61.73 & 67.44 & 72.74 \\
\hline FEB & 58.81 & 60.11 & 58.00 & 58.49 & 60.44 & 55.75 & 64.44 & 66.14 \\
\hline MAR & 60.04 & 63.11 & 62.56 & 63.48 & 64.19 & 68.87 & 68.64 & 70.37 \\
\hline APR & 63.20 & 61.15 & 60.01 & 59.10 & 60.78 & 61.59 & 65.78 & 72.78 \\
\hline MAY & 61.75 & 62.88 & 62.98 & 62.63 & 63.76 & 64.80 & 68.82 & 72.61 \\
\hline$\pi N$ & 58.35 & 60.25 & 61.42 & 59.65 & 61.31 & 59.20 & 66.06 & 72.55 \\
\hline$\pi R$ & 62.46 & 60.73 & 59.98 & 63.38 & 59.56 & 61.41 & 67.63 & 70.96 \\
\hline $\mathrm{AUG}$ & 60.85 & 59.12 & 62.34 & 62.97 & 48.87 & 62.97 & 69.93 & 71.23 \\
\hline SEP & 60.24 & 62.36 & 60.32 & 64.32 & 59.73 & 62.03 & 67.52 & 72.23 \\
\hline OCT & 61.59 & 63.25 & 62.47 & 62.47 & 64.65 & 64.78 & 70.18 & 74.69 \\
\hline NOV & 58.44 & 60.21 & 63.78 & 63.78 & 59.55 & 63.67 & 69.83 & 71.24 \\
\hline $\mathrm{DEC}$ & 65.34 & 64.32 & 66.32 & 67.42 & 61.64 & 66.66 & 72.83 & 71.77 \\
\hline TOTAL & 731.78 & 742.99 & 742.96 & 751.26 & 732.86 & 753.49 & 819.10 & 859.25 \\
\hline MEAN & 60.98 & 61.92 & 61.91 & 62.61 & 61.07 & 62.79 & 68.26 & 71.60 \\
\hline
\end{tabular}

TABLE 1 SALES OF PETROLEUM PRODUCTS (1996 - 2003) IN BILLION NAIRA

\section{DATA PRESENTATION AND ANALYSIS}

TABLE 2: SALES OF PETROLEUM PRODUCTS (MILLION NAIRA)

TABLE 3: QUARTERLY SALES OF PETROLEUM PRODUCTS (MILLION NAIRA)

\begin{tabular}{|l|l|l|l|l|l|l|}
\hline YEAR & QTR 1 & QTR 2 & QTR 3 & QTR 4 & TOTAL & MEAN \\
\hline 1996 & 179.56 & 183.30 & 183.55 & 185.37 & 731.78 & 182.95 \\
1997 & 188.72 & 184.28 & 182.21 & 187.78 & 742.99 & 185.75 \\
1998 & 183.34 & 184.41 & 182.64 & 192.57 & 742.96 & 185.74 \\
1999 & 185.55 & 181.38 & 190.67 & 193.67 & 751.27 & 187.82 \\
2000 & 193.01 & 185.85 & 168.16 & 185.84 & 732.86 & 183.22 \\
2001 & 186.38 & 185.59 & 186.41 & 195.11 & 753.49 & 188.37 \\
2002 & 200.52 & 200.66 & 205.08 & 212.84 & 819.10 & 204.78 \\
2003 & 209.25 & 217.94 & 214.42 & 217.64 & 859.25 & 214.81 \\
\hline
\end{tabular}


TABLE 4 ARRANGING THE QUARTERLY SALES DATA (IN MIILION NAIRA) IN SERIES

\begin{tabular}{|c|c|c|c|c|c|c|}
\hline $\mathrm{T}$ & $\begin{array}{c}\mathrm{K}=0 \\
y_{\mathrm{t}}\end{array}$ & $\begin{array}{c}\mathrm{K}=1 \\
y_{t+1}\end{array}$ & $\begin{array}{c}\mathrm{K}=2 \\
y_{t+2}\end{array}$ & $\begin{array}{c}\mathbf{K}=\mathbf{3} \\
y_{t+2}\end{array}$ & $\begin{array}{c}\mathrm{K}=4 \\
y_{t+2}\end{array}$ & $\begin{array}{c}\mathbf{K}=\mathbf{5} \\
y_{t+4}\end{array}$ \\
\hline 1 & 179.56 & 183.30 & 183.55 & 185.37 & 188.72 & 184.28 \\
\hline 2 & 183.30 & 183.55 & 185.37 & 188.72 & 184.28 & 182.21 \\
\hline 3 & 183.55 & 185.37 & 188.72 & 184.28 & 182.21 & 187.78 \\
\hline 4 & 185.37 & 188.72 & 184.25 & 182.21 & 187.78 & 183.34 \\
\hline 5 & 188.72 & 184.28 & 182.21 & 187.78 & 183.34 & 184.41 \\
\hline 6 & 184.28 & 182.21 & 187.78 & 183.34 & 184.41 & 182.64 \\
\hline 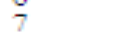 & 182.21 & 187.78 & 183.34 & 184.41 & 182.64 & 192.57 \\
\hline 8 & 187.78 & 183.34 & 184.41 & 182.64 & 192.57 & 185.55 \\
\hline 9 & 183.34 & 184.41 & 182.64 & 192.57 & 185.55 & 181.38 \\
\hline 10 & 184.41 & 182.64 & 192.57 & 185.55 & 181.38 & 190.67 \\
\hline 11 & 182.64 & 192.57 & 185.55 & 181.38 & 190.67 & 193.67 \\
\hline 12 & 192.57 & 185.55 & 181.38 & 190.67 & 193.67 & 193.01 \\
\hline 13 & 185.55 & 181.38 & 190.67 & 193.67 & 193.01 & 185.85 \\
\hline 14 & 181.38 & 190.67 & 193.67 & 193.01 & 185.85 & 168.16 \\
\hline is & 190.67 & 193.67 & 193.01 & 185.85 & 168.16 & 185.84 \\
\hline 16 & 193.67 & 193.01 & 185.85 & 168.16 & 185.84 & 186.38 \\
\hline 17 & 193.01 & 185.85 & 168.16 & 185.84 & 186.38 & 185.59 \\
\hline 18 & 185.85 & 168.16 & 185.84 & 186.38 & 185.59 & 186.41 \\
\hline 19 & 168.16 & 185.84 & 186.38 & 185.59 & 186.41 & 195.11 \\
\hline 20 & 185.84 & 186.38 & 185.59 & 186.41 & 195.11 & 200.52 \\
\hline 21 & 186.38 & 185.59 & 186.41 & 195.11 & 200.52 & 200.66 \\
\hline 22 & 185.59 & 186.41 & 195.11 & 200.52 & 200.66 & 205.08 \\
\hline 23 & 186.41 & 195.11 & 200.52 & 200.66 & 205.08 & 212.84 \\
\hline 24 & 195.11 & 200.52 & 200.66 & 205.08 & 212.84 & 209.25 \\
\hline 25 & 200.52 & 200.66 & 205.08 & 212.84 & 209.25 & 217.94 \\
\hline 26 & 200.66 & 205.08 & 212.84 & 209.25 & 217.94 & 214.42 \\
\hline 27 & 205.08 & 212.84 & 209.25 & 217.94 & 214.42 & 217.64 \\
\hline 28 & 212.84 & 209.25 & 217.94 & 214.42 & 217.64 & - \\
\hline 29 & 209.25 & 217.94 & 214.42 & 217.64 & - & - \\
\hline 30 & 217.94 & 214.42 & 217.64 & & - & - \\
\hline 31 & 214.94 & 217.64 & 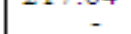 & - & - & - \\
\hline 32 & 217.64 & - & - & - & - & - \\
\hline TOTAL & 6133.70 & & & & & \\
\hline
\end{tabular}

ESTIMATION OF AUTOCOVARIANCE AND AUTOCORRELATION FOR LAGS K; 0, 1, 2, 3, 4 AUTO COVARIANCE $=\sum_{t=1}^{T-k} \frac{\left(y_{t}-y_{1}\right)\left(y_{t-k}-y_{t}\right)}{T}$

$$
\begin{aligned}
& \text { VARIANCE } \\
& \text { MEAN }
\end{aligned}=\sum \frac{y_{t}}{T}=\sum_{t=1}^{T-k}\left(y_{t}-y_{1}\right)^{2}
$$

\begin{tabular}{|c|c|c|c|c|c|}
\hline & & \multicolumn{2}{|c|}{$\mathrm{K}=0$} & \multicolumn{2}{|c|}{$\mathrm{K}=1$} \\
\hline $\mathrm{T}$ & $Y_{t}$ & $Y_{t}-y_{t}$ & $\left(V_{\mathrm{t}}-y_{t}\right)^{2}$ & $Y_{t-k}-y_{t}$ & $\left(Y_{t}-y_{t}\right)\left(V_{t-k}-y_{t}\right)$ \\
\hline 1 & 179.56 & -12.12 & 146.89 & -8.38 & 101.57 \\
\hline 2 & 183.30 & -8.38 & 70.22 & -8.13 & 68.13 \\
\hline 3 & 183.55 & -8.13 & 66.10 & -6.31 & 51.30 \\
\hline 4 & 185.37 & -6.31 & 39.82 & -2.96 & 81.68 \\
\hline 5 & 188.72 & -2.96 & 8.76 & -7.40 & 21.90 \\
\hline 6 & 184.28 & -7.40 & 54.76 & -9.47 & 70.08 \\
\hline 7 & 182.21 & -9.47 & 89.68 & -3.90 & 36.93 \\
\hline 8 & 187.78 & -3.90 & 15.21 & -8.34 & 32.53 \\
\hline 9 & 183.34 & -8.34 & 69.59 & -7.27 & 60.63 \\
\hline 10 & 184.41 & -7.27 & 52.85 & -9.04 & 65.72 \\
\hline 11 & 182.64 & -9.04 & 81.72 & 0.59 & -8.05 \\
\hline 12 & 192.57 & 0.89 & 0.79 & -6.13 & -5.46 \\
\hline 13 & 185.55 & -6.13 & 37.58 & -10.30 & 63.14 \\
\hline 14 & 181.38 & -10.30 & 106.09 & -1.01 & 10.40 \\
\hline 15 & 190.67 & -1.01 & 1.02 & 1.99 & -2.01 \\
\hline 16 & 193.67 & 1.99 & 3.96 & 1.33 & 2.65 \\
\hline 17 & 193.01 & 1.33 & 1.77 & -5.83 & -7.75 \\
\hline 18 & 185.85 & -5.86 & 33.99 & -23.52 & 137.12 \\
\hline 19 & 168.16 & -23.52 & 553.19 & -5.84 & 137.36 \\
\hline 20 & 185.84 & -5.84 & 34.11 & -5.30 & 30.95 \\
\hline 21 & 186.38 & -5.30 & 28.09 & -6.09 & 32.28 \\
\hline 22 & 185.59 & -6.09 & 37.09 & $\begin{array}{r}-5.27 \\
3.43\end{array}$ & 32.09 \\
\hline 23 & 186.41 & -5.27 & 27.77 & $\begin{array}{l}3.43 \\
8.84\end{array}$ & -18.08 \\
\hline $\begin{array}{l}24 \\
25\end{array}$ & $\begin{array}{l}195.11 \\
200.52\end{array}$ & $\begin{array}{l}3.43 \\
8.84\end{array}$ & $\begin{array}{l}11.76 \\
78.15\end{array}$ & $\begin{array}{l}8.84 \\
8.98\end{array}$ & $\begin{array}{r}30.32 \\
79.38\end{array}$ \\
\hline 26 & 200.66 & 8.98 & 80.64 & 13.40 & 120.33 \\
\hline 27 & 205.08 & 13.40 & 179.56 & 21.16 & 283.54 \\
\hline 28 & 212.84 & 21.16 & 447.75 & 17.57 & 371.78 \\
\hline 29 & 209.25 & 17.57 & 308.79 & 26.26 & 461.39 \\
\hline 30 & 217.94 & 26.26 & 689.59 & 22.74 & 597.15 \\
\hline 31 & 214.42 & 22.74 & 517.11 & 25.96 & 590.33 \\
\hline 32 & 217.42 & 25.96 & 673.92 & - & - \\
\hline TOTAL & 6133.70 & & 4548.20 & & 3466.33 \\
\hline
\end{tabular}

$\mathrm{T} \quad=$ No of observation in the series

TABLE5: Estimation of auto covariance and autocorrelation for lag $k, 0,1,2,3,4$. 


\begin{tabular}{|c|c|c|c|c|}
\hline & & $\mathrm{K}=\mathbf{2}$ & $\mathrm{K}=\mathbf{3}$ & \\
\hline $\mathrm{T}$ & $Y_{t+k}-y_{t}$ & $\left(Y_{t}-y\right)\left(Y_{t+k}-y\right)$ & $Y_{t-k}-y_{t}$ & $\left(y_{t}-y\right)\left(Y_{t k}-y_{)}\right)$ \\
\hline 1 & -8.13 & 98.54 & -6.31 & 76.48 \\
\hline 2 & -6.31 & 52.88 & -2.96 & 24.80 \\
\hline 3 & -2.96 & 24.06 & -7.40 & 60.16 \\
\hline 4 & -7.40 & 46.69 & -9.47 & 59.76 \\
\hline 5 & -9.47 & 28.03 & -3.90 & 11.54 \\
\hline 6 & -3.90 & 28.86 & -8.34 & 61.72 \\
\hline 7 & -8.34 & 78.98 & -7.27 & 68.85 \\
\hline 8 & -7.27 & 28.35 & -9.04 & 35.26 \\
\hline 9 & -9.04 & 72.39 & 0.89 & -7.42 \\
\hline 10 & 0.89 & -6.47 & -6.13 & 44.57 \\
\hline 11 & -6.13 & 55.42 & -10.30 & 93.11 \\
\hline 12 & -10.30 & -9.17 & -1.01 & -0.90 \\
\hline 13 & -1.01 & 6.19 & 1.99 & -12.20 \\
\hline 14 & 1.99 & -20.50 & 1.33 & -13.70 \\
\hline 15 & 1.33 & -1.34 & -5.83 & -5.89 \\
\hline 16 & -5.83 & -11.60 & -23.52 & -46.80 \\
\hline 17 & -23.52 & -31.28 & -5.84 & -7.77 \\
\hline 18 & -5.84 & 34.05 & -5.30 & 30.89 \\
\hline 19 & -5.30 & 124.66 & -6.09 & 143.24 \\
\hline 20 & -6.09 & 35.57 & -5.27 & 30.78 \\
\hline 21 & -5.27 & 27.93 & 3.43 & -18.18 \\
\hline 22 & 3.43 & -20.89 & 8.84 & -53.84 \\
\hline 23 & 8.84 & -46.59 & 8.98 & -47.32 \\
\hline 24 & 8.98 & 30.80 & 13.40 & 45.96 \\
\hline 25 & 13.40 & 118.46 & 21.16 & 187.05 \\
\hline 26 & 21.16 & 190.02 & 17.57 & 157.78 \\
\hline 27 & 17.57 & 235.44 & 26.26 & 351.88 \\
\hline 28 & 26.26 & 555.66 & 22.74 & 481.18 \\
\hline 29 & 22.74 & 399.54 & 25.96 & 456.12 \\
\hline 30 & 25.96 & 681.71 & - & - \\
\hline 31 & - & - & - & - \\
\hline \multirow[t]{2}{*}{32} & - & - & - & - \\
\hline & - & 2806.39 & - & 2218.89 \\
\hline
\end{tabular}

\begin{tabular}{|c|c|c|c|c|}
\hline & & $K=4$ & $=5$ & \\
\hline $\mathrm{T}$ & $Y_{t k}-y_{t}$ & $\left(Y_{t}-y_{0}\right)\left(Y_{t k}-y\right)$ & $Y_{t k}-y_{t}$ & $\left(y_{t}-y\right)\left(Y_{r k}-y\right)$ \\
\hline 1 & -2.96 & 35.88 & -7.40 & 89.69 \\
\hline 2 & -7.40 & 62.01 & -9.47 & 79.36 \\
\hline 3 & -9.47 & 76.99 & -3.90 & 31.71 \\
\hline 4 & -3.90 & 24.61 & -8.34 & 52.63 \\
\hline 5 & -8.34 & 24.69 & -7.27 & 21.54 \\
\hline 6 & -7.27 & 53.80 & -9.04 & 66.90 \\
\hline 7 & -9.04 & 85.61 & 0.89 & -8.43 \\
\hline 8 & 0.89 & -3.47 & -6.13 & 23.91 \\
\hline 9 & -6.13 & 51.12 & -10.30 & 85.90 \\
\hline 10 & -10.30 & 74.88 & -1.01 & 7.34 \\
\hline 11 & -1.01 & 9.13 & 1.99 & -17.99 \\
\hline 12 & 1.99 & 1.77 & 1.33 & 1.18 \\
\hline 13 & 1.33 & -8.15 & -5.83 & 35.74 \\
\hline 14 & -5.83 & 60.05 & -23.52 & 242.26 \\
\hline 15 & -23.52 & 23.76 & -5.84 & -10.55 \\
\hline 16 & -5.84 & -11.62 & -5.30 & -8.10 \\
\hline 17 & -5.30 & -7.05 & -6.09 & 30.72 \\
\hline 18 & -6.09 & 35.50 & -5.27 & -80.67 \\
\hline 19 & -5.27 & 123.95 & 3.43 & -51.63 \\
\hline 20 & 3.43 & -20.03 & 8.84 & -47.59 \\
\hline 21 & 8.84 & -46.85 & 8.98 & -81.61 \\
\hline 22 & 8.98 & -54.69 & 13.40 & -111.51 \\
\hline 23 & 13.40 & -70.62 & 21.16 & 60.27 \\
\hline 24 & 21.16 & 72.78 & 17.57 & 232.14 \\
\hline 25 & 17.57 & 155.32 & 26.26 & 249.11 \\
\hline 26 & 26.26 & 235.81 & 27.74 & 347.86 \\
\hline 27 & 22.74 & 304.72 & 25.96 & - \\
\hline 28 & 25.96 & 549.31 & - & - \\
\hline 29 & - & - & - & - \\
\hline 30 & - & - & - & - \\
\hline 31 & - & - & - & - \\
\hline 32 & - & - & - & - \\
\hline TOTAL & 1839.21 & - & 1246.06 & \\
\hline
\end{tabular}


Let the variable be denoted by

\section{COMPUTATIONS}

$\Upsilon_{0}=\quad \frac{4548.20}{32}=142.13$

The auto covariance of lag $\mathrm{k}$ is $\Upsilon_{\mathrm{k}}$ for $\mathrm{k}=1,2,3,4$ and 5

$\Upsilon_{0}=1 \quad=\frac{3466.32}{32}=108.32$

$\Upsilon_{0}=2=\underline{2806.39}=87.10$

$\Upsilon_{0}=3=\frac{2218.89}{32} \quad=69.34$

$\Upsilon_{0}=4=\frac{1839.21}{32} \quad=57.48$

$\Upsilon_{0}=5=\frac{1246.06}{32}=38.94$

Denote the autocorrelation by $\mathrm{P}_{\mathrm{k}}$ and given by

$\mathrm{P}_{\mathrm{k}} \quad=\quad \Upsilon_{\mathrm{k}}, \quad$ for $\mathrm{k}=0,1,2,3,4$ and 5

$\mathrm{P}_{\mathrm{k}}=0=\frac{142.13}{32}=1$

$\mathrm{P}_{\mathrm{k}}=1=\frac{108.32}{32}=1$

$\mathrm{P}_{\mathrm{k}}=2=\frac{87.10}{32}=0.61$

$\mathrm{P}_{\mathrm{k}}=3=\frac{69.34}{32}=0.49$

$\mathrm{P}_{\mathrm{k}}=4=\frac{57.48}{32}=0.46$

$\mathrm{P}_{\mathrm{k}}=5=\frac{38.94}{32}=0.27$

TABLE 6 ARRANGEMENT OF AUTOCOVARIANCE AND AUTOCORELATION

\begin{tabular}{|l|l|l|l|l|l|l|}
\hline K & $\mathbf{0}$ & $\mathbf{1}$ & $\mathbf{2}$ & $\mathbf{3}$ & $\mathbf{4}$ & $\mathbf{5}$ \\
\hline Auto Covariance & 142.13 & 108.32 & 87.10 & 67.34 & 57.48 & 38.94 \\
\hline Auto Correlation & 1 & 0.76 & 0.61 & 0.49 & 0.40 & 0.27 \\
\hline
\end{tabular}

ESTIMATING THE PARAMETER $\phi_{1} \& \phi_{2}$ FOR THE AUTOREGRESSIVE PROCESS

Recall

$$
\mathrm{P}_{1}=0.76 \mathrm{P}_{2}=0.61
$$

$\phi_{1}+\phi_{2} \mathrm{P}_{1}=\mathrm{P}_{1}$

$\phi_{1} \mathrm{P}_{1}+\phi_{2}=\mathrm{P}_{2}$

Substituting the values of $\mathrm{P}$ above into the equation

$\phi_{1}+0.76 \phi_{2}=0.76$

$0.76 \phi_{1}+\phi_{2}=$

0.61

Multiply equ.

by 0.76

(2) by 1

$0.76 \phi_{1}+0.58 \phi_{2}=0.58$

$0.76 \phi_{1}+\phi_{2}=\quad 0.61$

Subtract (4) from (3)

$-0.58 \phi_{2}=-0.03$

From equ (5)

$\phi_{2}=0.05$

Substitute $\phi_{2} \quad=0.05$ in equ (1)

$\phi_{1}+0.76(0.05)=0.76$ 


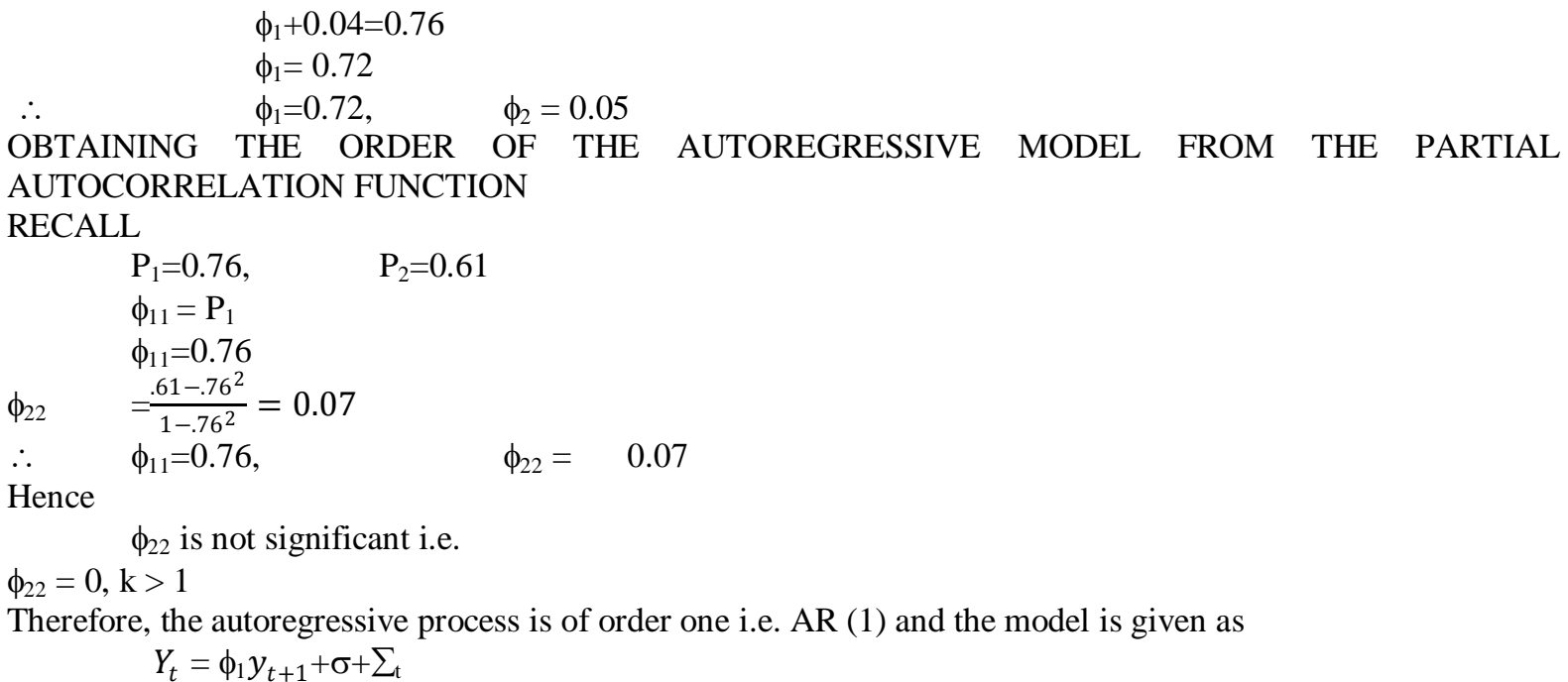

Assuming $\sum_{\mathrm{t}}$ and $\sigma$ to be zero since the series is stationary as observed in the sample autocorrelation. Thus, the autoregressive model for this process can be presented as

Where

$$
Y_{t+1}=\phi_{1} y_{t}
$$

NOTE

$$
Y_{t} \Rightarrow \text { Last figure in the series }
$$

$$
\phi_{1}=0.76, \quad y_{t}=217.64
$$

FORECASTING THE AUTORESSIVE PROCESS AR (1) FOR 2004 QUARTERS FOR FIRST QUARTER

$$
\begin{array}{lc}
Y_{t+1}= & \phi_{1} y_{t} \\
=0.76 \times 217.64 \\
Y_{t+1}=\quad 165.41
\end{array}
$$

FOR SECOND QUARTER

$$
\begin{array}{rll}
Y_{t+2}= & \Upsilon \phi_{1} y_{t+1} & \\
& = & 0.76 \times 165.41 \\
& = & 125.71
\end{array}
$$

FOR THIRD QUARTER

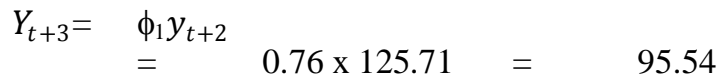

FOR FOURTH QUARTER

$$
\begin{aligned}
& Y_{t+4}=\Upsilon \phi_{1} y_{t+3} \\
& =0.76 \times 95.61=72.66 \\
& =92.66
\end{aligned}
$$

FORECASTED FIGURES FOR 2004 QUARTERS

$\begin{array}{lll}\text { QTR1 } & & 165.41 \\ \text { QTR2 } & & 125.71 \\ \text { QTR3 } & = & 95.61 \\ \text { QTR4 } & = & 72.66\end{array}$

\section{FINDINGS}

1 Fluctuations in sales are mostly apparent in ' 96 and ' 98 . This might be due to lack of adequate supply of stock to the depot.

2 Sales at its peak between '01 and '03. This might

Be due to sharp increase of prices of petroleum produce. The series witness stationarity for the years under study. This was as the sample autocorrelation

3 The forecast for the series is best obtained at autoregressive model of order- one i.e. AR(1)

4The forecasted values for 2004 quarters were

Observed to be less than the observed values for the previous year. More also, as the forecast for 2004 Quarters were increasing in the same order, the forecasted values were decreasing. This simply explains the 
standpoint of the exorbitant prices and the mind of the consumers. This is to say, there might be general fall in sales in future if the consumers have their way.

\section{RECOMMENDATION}

1. Community and labour union disturbance which From time to time abrupt crude oil product and sales of refined products should be resolved by federal government in order to ensure a violent free environment for the company's operations.

2. The mode of operation in most of the section at the Depot is still very obsolete government should therefore, computerize the various section in the depot to enhance efficiency. More also, emphasis should be place on data management in order to ease assessment of the depot at any point in time.

3 The concept of mono-product economy should be de-emphasized. In other words federal government should from now attend to agriculture, and solid minerals, this would help to diversify employment base and foreign exchange earnings

\section{REFERENCES}

[1] Asanya E.O Unpublished note on Time Series Analysis (I.M.T) Enugu

[2] Augustine O.O Petroleum and the Nigeria Economy, Africa Top Publisher Ltd

[3] Anderson O.D. Time Series Analysis and Forecasting (The Box - Jenkins) approach) the Butter Worth Group

[4] Devore, J.and Peck, R. (1986)

[5] statistics: the exploration and analysis of data.London.west publishing co.ltd John N.Igabari (2012). A Functional Approach to Statistics Amekatik Research Publishers. Uche P.1 (2003).

[6] Probability and applications. Longman Nigeria Plc. Forecasting (The Box - Jenkins) approach) the Butter Worth Group Spiegel, M.R. (1990)

[7] Schaums outline series: statistics. New York. McGraw Hill Book Co. Walpole, R.E (1982).

[8] Introduction to statistics. $3^{\text {rd }}$ edition New York Macmillan Publishing Company Inc. Warren Gilchrist Statistical Forecasting a Willey - Interscience Publications

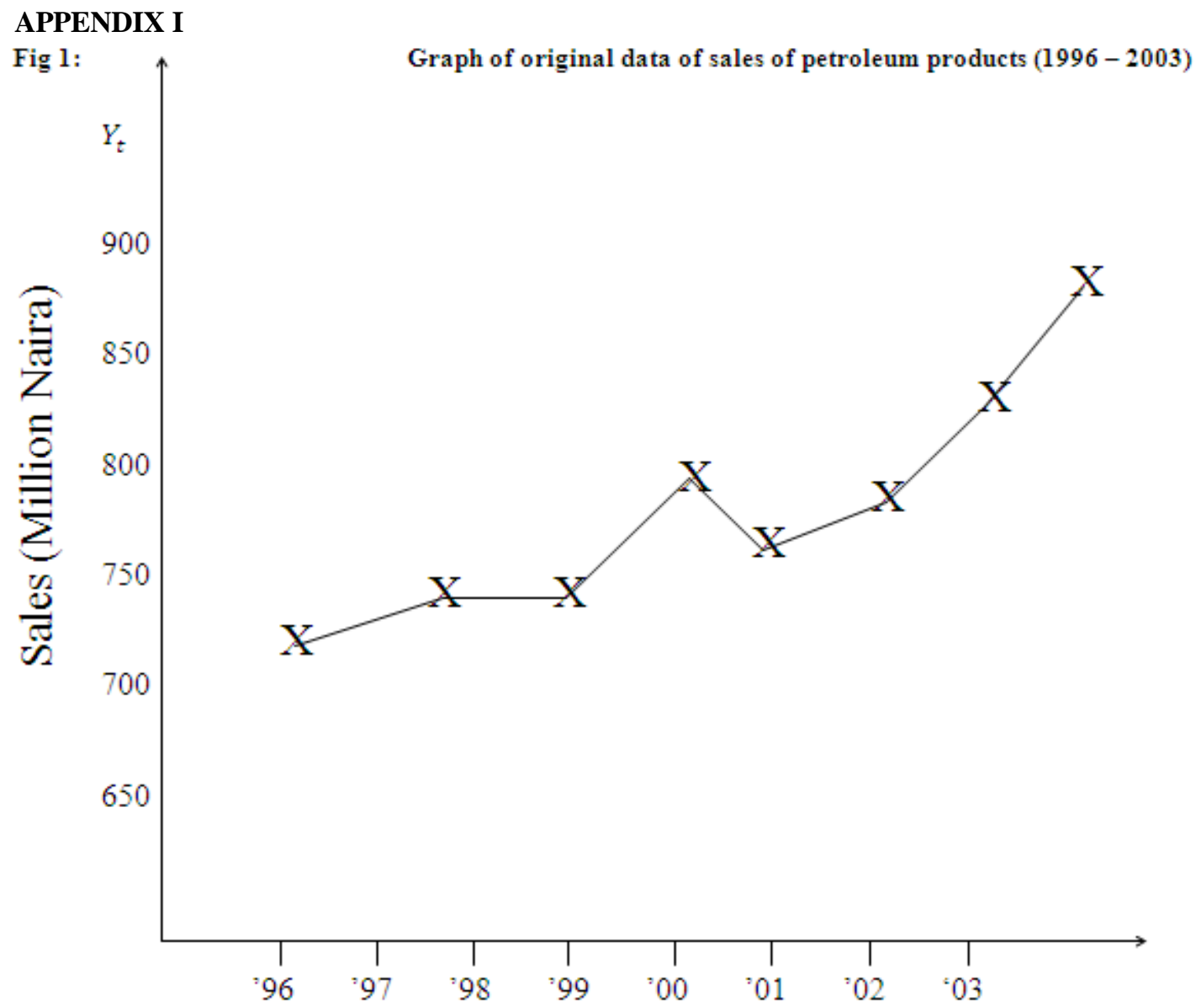




\section{APPENDIX II}

Fig 2GRAPH OF THE SAMPLE AUTOCORRELATION FUNCTION (COROLELO GRAM)

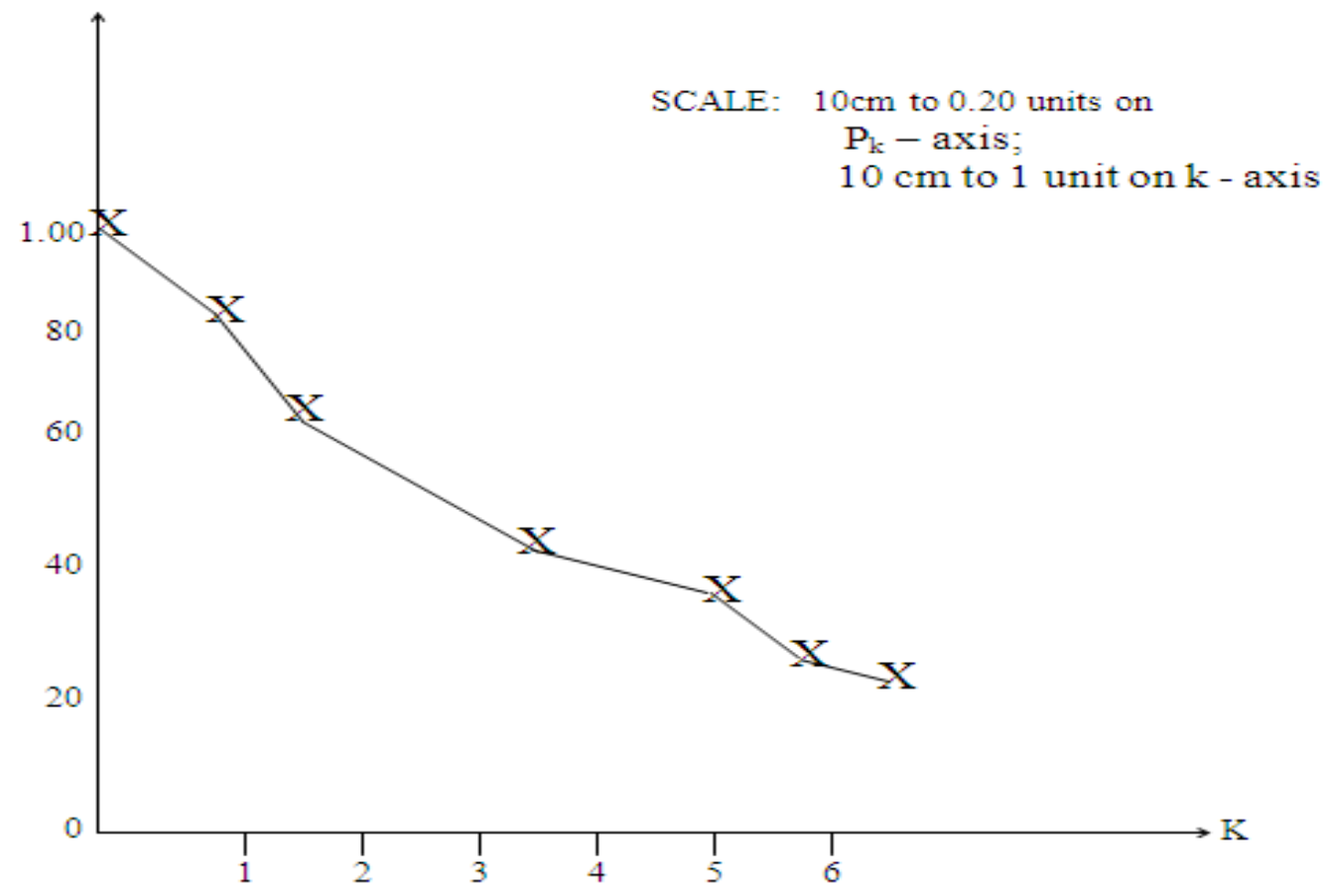

